\title{
On the FPA infrared camera transfer function calculation
}

\author{
by S. Datcu ${ }^{1}$, L. Ibos ${ }^{1}, Y$. Candau $^{1}$, S. Matteï ${ }^{2}$ \\ (1) CERTES, Université Paris XII Val de Marne, Créteil, France \\ (2) LTM, Université de Bourgogne, Le Creusot, France
}

\begin{abstract}
In order to obtain a good quantification of the heat flux emitted by a surface using infrared images, it is necessary to calculate accurate values of the surface temperature. Infrared images often distorted due to aberration and diffraction phenomena and electronic noise. A convolution product can model aberration and diffraction distortions and the detector noise is assumed to be stationary and additive. Image restoration can be treated as an ill-posed problem. Its solution is commonly obtained using regularisation methods.
\end{abstract}

\section{Introduction}

The physical approach used in this work is to study the correspondence between all surface elements of an object $\mathrm{M}$ observed by an infrared camera and their images in the image plane (i.e. the detector matrix). The luminance of each surface element $\mathrm{M}_{0}$ of coordinates $\left(x_{o}, y_{o}\right)$ can be represented in the object plane by a two-dimension Dirac distribution $\delta\left(\mathrm{x}_{0}-x, \mathrm{y}_{0}-y\right)$. The image of the element $\mathrm{M}_{0}$ through an optical system is a blur image located around the corresponding elements $M_{0}^{\prime}\left(x_{0}^{\prime}, y_{0}^{\prime}\right)$ in the image plane. The irradiance distribution of this image can be represented by the impulse response $h$, also called the point-spread function (PSF). This function is often considered as position invariant in a limited area of the image plane [1,2].

Considering an object $M$ with a luminance distribution $L_{o}(x, y)$, the irradiance distribution $E\left(x^{\prime}, y^{\prime}\right)$ in the image plane is given by the convolution product between $L_{0}$ and the impulse response $h[3]$ :

$$
E\left(x^{\prime}, y^{\prime}\right)=L_{o}(x, y) * h\left(x^{\prime}, y^{\prime}\right)
$$

The spectrum of the impulse response $h\left(x^{\prime}, y^{\prime}\right), H(\xi, \eta)=\mathfrak{I} \mathfrak{I}\left(h\left(x^{\prime}, y^{\prime}\right)\right)$ is the transfer function of the infrared viewing system; $\mathfrak{I} \mathfrak{I}$ is the two-dimensional Fourier transform operator and $\xi, \eta$ are spatial frequencies in the Fourier space.

The infrared systems consist of several subsystems, each with is own impulse response and the final result of all the subsystems operating on the input object distribution is the multiplication of their respective transfer functions. We assume that $H(\xi, \eta)$ has been normalized to have unit value at zero spatial frequency. This normalization yields a relative transmittance for the various frequencies and ignores attenuation factors that are independent of spatial frequency. With this normalization, $H(\xi, \eta)$ is referred as the optical transfer function (OTF) which is generally a complex function having both a magnitude (the modulation transfer function - MTF) and a phase (phase transfer function - PTF) portion:

$$
\mathrm{OTF}=H(\xi, \eta)=|H(\xi, \eta)| \cdot e^{(-j \cdot \theta(\xi, \eta))}=\operatorname{MTF}(\xi, \eta) \cdot e^{(-j \cdot P T F(\xi, \eta))}
$$


The aim of this work is to determinate the overall transfer function of an infrared camera system. In the case of an infrared system of focal plane array (FPA) detectors, it consist of four components [2]: the diffraction transfer function $O T F_{\text {diffraction }}$, the geometrical transfer function $O T F_{\text {geometrical }}$, the footprint detector transfer function $O T F_{\text {footprint }}$ and the sampling transfer function OTF $_{\text {sampling }}$.

\subsection{Diffraction OTF}

Because of the wave nature of light, an optical system with a finite-sized aperture can never form a point image. Their characteristic minimum blur diameter, when other defects (aberrations) are absent, is [2]:

$$
d_{\text {diffraction }}=2.44 \cdot \lambda \cdot F / D
$$

where $F$ is the distance from the lens to the object plane and $D$ is the aperture size of the lens. Conceptually, the diffraction OTF can be calculated as the magnitude of the Fourier transform of the diffraction impulse response profile. Also, the diffraction OTF can be calculated as the normalized autocorrelation of the exit pupil of the system [1,2]. For incoherent systems, the impulse response $h(x, y)$ is the square of the two-dimensional Fourier transform of the diffracting aperture $p(x, y)$ :

$$
h_{\text {diffraction }}(x, y)=|\mathfrak{I} \mathfrak{I}(p(x, y))|^{2}
$$

with:

$$
p(x, y)=\operatorname{rect}(x / D, y / D)
$$

Then, the diffraction OTF is given by:

$$
\operatorname{OTF}_{\text {diffraction }}(\xi, \eta)=\mathfrak{I} \mathfrak{I}\left(h_{\text {diffraction }}(x, y)\right)
$$

\subsection{Geometrical OTF}

Considering first the one-dimensional case. A point source of light, of wavelength $\lambda$, is located at infinity along the optical axis, from which spherical waves emanate. At an infinite distance from the lens they appear as planes waves, as illustrated at the far left of figure 1.

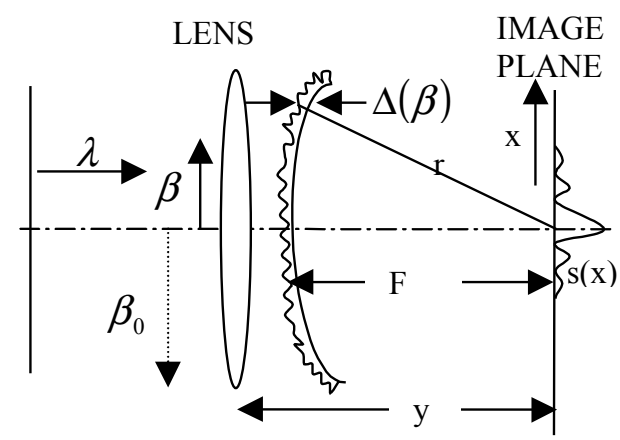

Fig. 1. Image formation physics 
At each coordinate $\beta$ of the pupil, there is a phase distance error $\Delta$ from a sphere with radius $\mathrm{F}$ (i.e., which perfectly focuses light in the image plane). The lens aperture size $\beta_{0}$ is assumed to be small enough to allow Huygen's scalar theory of light to be used. Huygen's principle states that each point $\beta$ of the wave front just to the right of the lens acts like a new source of spherical waves. This defines a complex "pupil function" $e^{j \cdot k \cdot \Delta(\beta)}$ at each point $\beta$ of the pupil [1] ( $k=2 \cdot \pi / \lambda$ is the wave number). The focused image has an intensity profile $h(x)$ (the point spread function) which is located in the image plane. This may be represented in terms of a point amplitude function $a(x)$ as:

$$
h(x)=|a(x)|^{2}
$$

Quantity $a(x)$ relates to the pupil function by Fraunhofer's integral:

$$
a(x)=\int_{-\beta_{0}}^{\beta_{0}} d \beta \cdot e^{[j \cdot(k \cdot \Delta(\beta)-\beta \cdot x)]}
$$

which is basically a finite Fourier transform of the pupil function. Finally, the optical transfer function $\operatorname{OTF}(\xi)$ is the Fourier transform of $h(x)$.

Extending this result to the two-dimensional case, we obtain:

$$
a(x, y)=\mathfrak{I} \mathfrak{I}\left(e^{j \cdot k \cdot \Delta\left(\beta_{1}, \beta_{2}\right)}\right) \quad h(x, y)=|a(x, y)|^{2} \quad \operatorname{OTF}_{\text {geometrical }}(\xi, \eta)=\mathfrak{I} \mathfrak{I}(h(x, y))
$$

Considering that the optical pupil is generally aberrated, with a pupil function $U\left(\beta_{1}, \beta_{2}\right)=e^{j \cdot \Phi\left(\beta_{1}, \beta_{2}\right)}$, where $\Phi\left(\beta_{1}, \beta_{2}\right)$ is the aberration function [1] and supposing that this function can have a third degree polynomial form depending on the pupil coordinates $\left(\beta_{1}, \beta_{2}\right)$ :

$$
\Phi\left(\beta_{1}, \beta_{2}\right)=a_{1} \cdot \rho+a_{2} \cdot \rho^{2}+a_{3} \cdot \rho^{3}, \quad \rho^{2}=\beta_{1}^{2}+\beta_{2}^{2}
$$

This first assumption implies that the irradiance distribution on the aberration blur area is approximated by the most likely diffraction irradiance distribution and the second one allows to predict the evolution of the equivalent diffraction blur on the image plane. Then, the geometrical impulse response has a parametrical form:

$$
h=h\left(x, y, \Phi\left(\beta_{1}, \beta_{2}\right)\right)=h\left(x, y, a_{i}\right)
$$

\subsection{Detector footprint OTF}

The footprint of a particular detector is its projection onto the object space. If we consider a FPA imaging system, the flux falling onto an individual detector produces a single output which is some spatial averaging of the image irradiance on the corresponding footprint surface. The integration of the scene irradiance $E(x, y)$ over the detector surface (considered as a square of side $w$ ) is equivalent to a convolution of $L_{O}(x$, $y)$ and the "rect" function that describe the detector responsivity:

$$
E\left(x^{\prime}, y^{\prime}\right)=\int_{-w / 2}^{w / 2} \int_{-w / 2}^{w / 2} L_{o}\left(x-x^{\prime}, y-y^{\prime}\right) d x d y=L_{o}\left(x^{\prime}, y^{\prime}\right) * \operatorname{rect}\left(x^{\prime} / w, y^{\prime} / w\right)
$$

By Fourier transformation of the impulse response $h\left(x^{\prime}, y^{\prime}\right)=\operatorname{rect}\left(x^{\prime} / w, y^{\prime} / w\right)$ we obtain the OTF, which is a two-dimensional "sinc" function: 


$$
\operatorname{OTF}_{\text {footpr int }}(\xi, \eta)=\sin c(\xi \cdot w, \eta \cdot w)
$$

\subsection{Sampling OTF}

A sampled-imaging system is not shift invariant [2]. The position of the imageirradiance function with respect to the sampling sites will affect the final image data. If we measure the OTF for such a sampled system, the Fourier transform of the spatial-domain image will depend on the alignment of the target and the sampling sites. The shift variance violates one of the main assumptions required for a convolutional analysis of the imageforming process. A spatially averaged impulse response and the corresponding OTF component must be defined by assuming that the scene being imaged is randomly positioned with respect to sampling sites. For a two-dimensional sampling grid, the sampling impulse response is a rectangle function of widths equal to the sampling intervals in each direction:

$$
h_{\text {sampling }}=\operatorname{rect}\left(x / x_{\text {samp }}, y / y_{\text {samp }}\right)
$$

and the OTF is defined by the Fourier transform of $h_{\text {sampling }}(x, y)$ :

$$
\operatorname{OTF}_{\text {sampling }}(\xi, \eta)=\mathfrak{I}\left(\operatorname{rect}\left(x / x_{\text {samp }}, y / y_{\text {samp }}\right)\right)=\sin c\left(\xi \cdot x_{\text {samp }}, \eta \cdot y_{\text {samp }}\right)
$$

\section{Experimental set-up}

Some of the major technical specifications of the camera used in this study (model AGEMA 570 Elite from FLIR Systems ${ }^{\mathrm{TM}}$ ) are listed in table 1. The experimental set-up is schematically presented in the figure 2 .

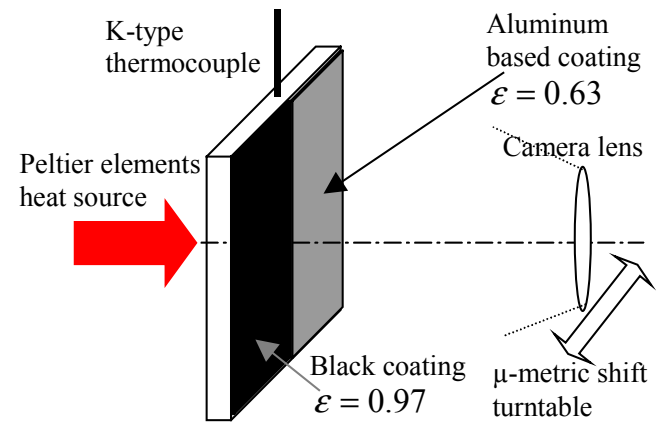

Fig. 2. Experimental set-up for transfer function camera measurement

The target scene is made of a $45 \times 45 \times 5 \mathrm{~mm}$ duralumin plate. In order to create an infrared knife-edge source, the viewed face of the plate was split in two equal vertical surfaces. One surface was coated with a black paint with emissivity 0.97 , while the other one was coated with an aluminum paint with emissivity 0.63 . The coatings used are very diffusive. This prevents any specular reflections of infrared external source. The Duralumin plate is heated by Peltier elements which allows to keep a stable temperature of the plate during measurements. The temperature of the plate is controlled by a K-type thermocouple. A fuzzy logic controller programmed under LabView ${ }^{\mathrm{TM}}$ regulates the heat flux supplied by the Peltier elements. The measurement of the transfer function of the camera was made for a fixed distance of $54.5 \mathrm{~cm}$ between the camera lens and the target 
scene. The acquisition of thermograms was made at a rate of about 7 images/second. The object parameters imposed to the camera during acquisition are listed in table 2 . The camera was placed on a turntable which allowed micro-metric step translations with a total length of $2.5 \mathrm{~cm}$. The step accuracy is $10 \mu \mathrm{m}$.

Table 1. Technical specifications of the Agema 570 camera

\begin{tabular}{|c|c|c|c|c|}
\hline $\begin{array}{c}\text { Field of } \\
\text { view }\end{array}$ & IFOV & $\begin{array}{c}\text { Spectral } \\
\text { range }\end{array}$ & Accuracy & Image size \\
\hline $45^{\circ} \times 34^{\circ}$ & $2.45 \mathrm{mrad}$ & $7.5-13 \mu \mathrm{m}$ & $\pm 2 \%$ of range or $\pm 2^{\circ} \mathrm{C}$ & $320 \times 240$ pixels \\
\hline
\end{tabular}

Table 2. Object parameters used for infrared images acquisition

\begin{tabular}{|c|c|c|c|c|c|}
\hline $\begin{array}{c}\text { Relative } \\
\text { Humidity }\end{array}$ & $\begin{array}{c}\text { Distance } \\
\text { Camera-Object }\end{array}$ & $\begin{array}{c}\text { Ambient } \\
\text { Temperature }\end{array}$ & $\begin{array}{c}\text { Atmospheric } \\
\text { Temperature }\end{array}$ & $\varepsilon$ & $\tau$ \\
\hline $50 \%$ & $54.5 \mathrm{~cm}$ & $20^{\circ} \mathrm{C}$ & $20^{\circ} \mathrm{C}$ & 1 & 1 \\
\hline
\end{tabular}

\section{Distortion model and identification procedure}

Generally, we can write:

$$
g\left(x^{\prime}, y^{\prime}\right)=\iint f(x, y) \cdot h\left(x^{\prime}-x, y^{\prime}-y, a_{i}\right) \cdot d x \cdot d y+n\left(x^{\prime}, y^{\prime}\right)
$$

where $g\left(x^{\prime}, y^{\prime}\right)$ is the irradiance distribution in the image plane, $f(x, y)$ is the luminance distribution in the object plane, $h\left(x^{\prime}-x, y^{\prime}-y, a_{i}\right)$ is the impulse response function of the imaging system and $n\left(x^{\prime}, y^{\prime}\right)$ is the noise (ergodic stationary Gaussian noise). The use of a lens with a large field of view implies a non-linear behavior of the camera optical system (i.e. the presence of lens aberrations). This behavior compelled us to define a multi-value impulsion response function in order to take this non-linearity into account. We can make a linear approximation of this problem by splitting the image in several areas where the camera response can be approximated by a convolution product. For each of these areas, a local impulse response can be defined (equation 11) and its values are given by the overall parametric PSF $h\left(x^{\prime}-x, y^{\prime}-y, a_{i}\right)$ where the parameters $a_{i}$ allow to compute the local value of the aberration function $\Phi\left(x^{\prime}, y^{\prime}\right)$ (equation 10 ).

Our approach to determine the camera transfer function is to use an infrared knifeedge source and to acquire the camera edge spread function (ESF) [2]. The ESF is the convolution of the PSF with the unit-step function:

$$
\operatorname{ESF}(x)=P S F(x, y) * \operatorname{step}(x)
$$

The camera response is noisy and thus we cannot solve the equation 17 for PSF by direct deconvolution because the deconvolution is an ill-posed problem [3]. In order to improve the signal-to-noise ratio, we averaged $N$ infrared images. Taking $N>30$, the central limit theorem allows us to consider a Gaussian distribution for the resulting noise [1] and thus, the variance of the mean resulting noise is $N$ times lower then the initial one. To avoid solving the problem by direct deconvolution, we proceeded by parametric identification. Because we cannot compute the PSF form ESF measurements, the parametric identification was performed for the amplitude portion of the PSF, the modulation transfer function (MTF). 


$$
\begin{aligned}
& \operatorname{PSF}_{k}(x, y)=h_{\text {diffraction }}(x, y) * h_{\text {geometrical }}^{k}(x, y) * h_{\text {footpr int }}(x, y) * h_{\text {sampling }}(x, y) \\
& i=1 . .3 \\
& \operatorname{MTF}_{k}(\xi, \eta)=\mathfrak{J I}\left(P S F_{k}(x, y)\right)
\end{aligned}
$$

where $k$ is the index of the area on the plane of view of the infrared camera. The diffraction, footprint and sampling PSF are known because we know the size of the aperture lens, the detector and the cell detector size. The geometrical PSF is the only unknown function. It is depending on the value of the aberration function $\Phi$. In a first step we will identified the $\Phi_{k}$ values on each of the measurement areas and in a second step we will compute the coefficients $a_{i}$ of the polynomial which fits the identified $\Phi_{k}$ values.

The "measured" values of the MTF used for fitting the analytical model are obtained from the ESF data [2] by first computing a line spread function (LSF) profile:

$$
\frac{d}{d x}(E S F(x))=\operatorname{LSF}(x)
$$

The magnitude of the one-dimensional Fourier transform of the line response provides information concerning one spatial-frequency component of the transfer function [2]:

$$
\mathfrak{I}(\operatorname{LSF}(x))=\operatorname{MTF}(\xi, 0)
$$

\section{Results}

Seven ESF were measured on seven position of the knife-edge source on the image plane. The thermogram obtained in the center of the field of view (FOV) is presented on figure 3.

For each position, the corresponding ESF was measured in about 20 points in order to obtain an accurate slope sampling of the ESF data (figure 4). Using equations 19 and 20 , we calculated the corresponding MTF components. The results are presented in figure 5 .

For each position, a $\Phi\left(\beta_{1}, \beta_{2}\right)$ value has been identified in order to obtain the best MTF fit. Two results of the fitting of the transfer function are presented in figure 6 .

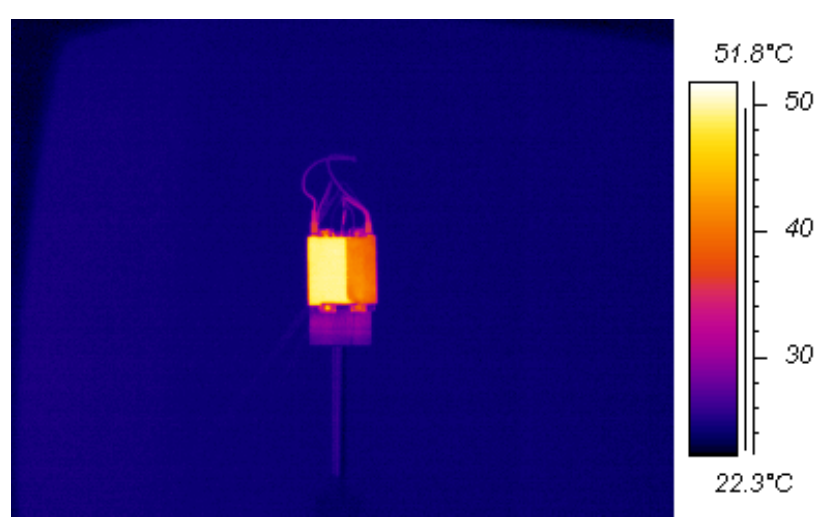

Fig. 3. Infrared image of the knife-edge source obtained in the center of FOV 


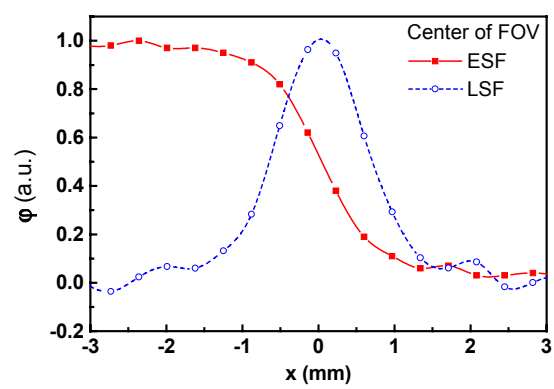

Fig. 4. Measured ESF and LSF in the center of FOV

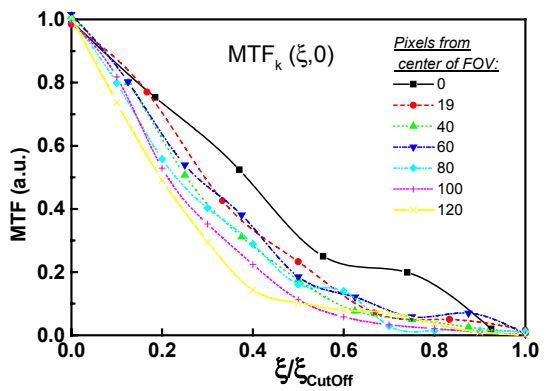

Fig. 5. Measured MTF at various position from the center of FOV

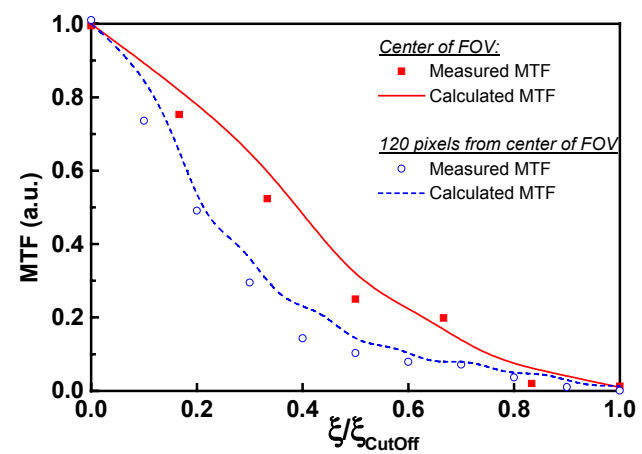

Fig. 6. MTF results in the FOV center and at 120 pixels from the FOV center

A good agreement between the "measured" and the calculated MTF component is observed, even on the proximity of the FOV limit of the camera (i.e. at 120 pixels from the FOV center - the extreme value is 160 pixels from the center). The values of the three parameters $a_{i}$ are obtained by least square fitting on the $\Phi\left(\beta_{1}, \beta_{2}\right)$ scalar values and we obtained the following values: $\left[\begin{array}{ll}-1.36 & 5.90 \cdot 10^{-1} \\ -2.05 \cdot 10^{-2}\end{array}\right]$. The residual norm of the fitting was low $\left(7.08 \cdot 10^{-7}\right)$ compared to values of $\Phi\left(\beta_{1}, \beta_{2}\right)$ of about 50 .

Two of the resulting PSFs, computed from the equation 18, are presented in the figure 7.

(a)

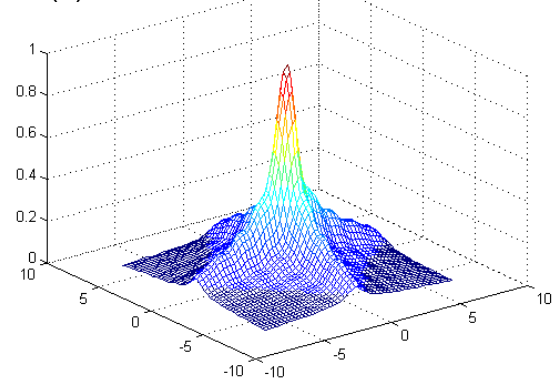

(b)

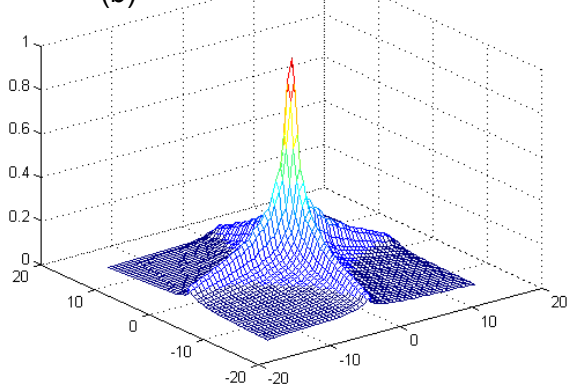

Fig. 7. PSF in the FOV center (a) and at 120 pixels from the FOV center (b) 


\section{Conclusion}

Obviously, the present model approximates locally the geometrical aberration blurs by the most likely diffraction blur (geometrically speaking). We obtained good MTF fit results that confirm the validity of this assumption in the studied configuration. The low value of the fit residuals validates the second assumption of the model: the size of the blur can be correlated with the position of the considered area center by a third degree polynomial. The next step will be image restoration by deconvolution with the PSF kernel obtained in this study.

\section{Acknowledgments}

This work was performed under the auspices of the R\&D Department of Electricité de France and we would like to thank the support of Jean-Claude Frichet.

\section{REFERENCES}

[1] FRIEDEN, B. R., "Probability, statistical optics, and data testing", Third Edition, Springer, Berlin 2001

[2] BOREMAN, Glenn D., "Modulation transfer function in optical and electro-optical systems", SPIE, 2001

[3] PAPINI, F. and GALLET, P., "Thermographie infrarouge", Masson, Paris 1990. 